\title{
Detailed Investigation of Silicon Nitride Phase Plates Prepared by Focused Ion Beam Milling
}

\author{
Alexander Müller ${ }^{1,2}$, Daniel B. Durham ${ }^{1,2}$, Karen C. Bustillo ${ }^{1}$, Frances I. Allen ${ }^{1,2}$ Andrew M. Minor ${ }^{1,2}$ \\ and Colin Ophus ${ }^{1, *}$ \\ 1. National Center for Electron Microscopy, Molecular Foundry, Lawrence Berkeley National \\ Laboratory, Berkeley, CA, USA. \\ 2. Department of Materials Science and Engineering, University of California, Berkeley, CA, USA. \\ *Corresponding author: cophus@gmail.com
}

By using SiN phase plates to shape the electron beam of transmission electron microscopes (TEM), results as diverse as vortex beams [1], phase contrast scanning TEM (STEM) [2], and sphericalaberration corrected STEM [3] have been achieved. To achieve a high performance, a phase plate must fulfill several requirements and its fabrication can be very challenging. The phase shift imposed on an electron beam depends on the thickness of the phase plate, and precise thickness control $(<1 \mathrm{~nm})$ with a high spatial resolution $(<10 \mathrm{~nm})$ is required over large areas (up to hundreds of $\mu \mathrm{m}^{2}$ ). The phase shift is further influenced by the chemical composition, necessitating clean processing conditions and that the fabrication does not influence the chemical composition in an unpredictable way. Lastly, the resulting phase plate should be as thin as possible to minimize high-angle scattering of the electron beam [4]. Focused ion beam (FIB) milling fulfills all of these requirements with a low time-investment and a high level of flexibility. It is therefore by far the most common phase plate preparation technique. However, several process parameters influence the quality of the resulting phase plate and optimizing them can be challenging and time-consuming. In this work, we analyze the effects of these process parameters on the milling process and discuss how our findings influence the performance of a phase plate.

To prevent charging, Norcada SiN membranes with a freestanding, $100 \mathrm{~nm}$ thick SiN film were coated with $\sim 2 \mathrm{~nm}$ of carbon. Squares with side lengths between 500 and $1000 \mathrm{~nm}$ were milled into SiN membranes using the $50 \mathrm{pA}, 30 \mathrm{kV}$ Ga-beam of a Zeiss ORION NanoFab multi-beam ion microscope. Typically, grids were set up with the total dose increasing in one direction and another parameter (dwell time, pixel spacing, mill order) varied in the other. The milled patterns were imaged using a FEI Titan TEM operated in STEM mode at an acceleration voltage of $60 \mathrm{kV}$. Energy-dispersive X-ray spectroscopy (EDS) maps were acquired using the attached Bruker SuperX EDS detector. We further measured height maps using a Bruker Icon atomic force microscope (AFM) operated in tapping mode.

As a first step, we determined the effects of the process parameters mill order, dwell time, and pixel spacing from STEM images. Using standard, line or serpentine mill orders resulted in a strongly sloped milled surfaces, presumably as a result of redeposition. In contrast, the double-serpentine mill order resulted in an evenly milled surface. Similarly, increasing the dwell time leads to increased redeposition and an uneven mill. In our tests, the shortest dwell times gave the best results. Finally, the pixel spacing should be chosen in accordance with the beam size. Very small pixel spacings resulted in uneven milling - essentially equivalent to increasing the dwell time - and high pixel spacings resulted in overmill, and the formation of a distinct raster pattern.

Having optimized the process parameters, we turned to a detailed analysis including high-resolution STEM images, AFM profiles and EDS maps. The surface roughness was comparable to the unmilled 
SiN membrane, permitting even the most demanding phase plate applications. However, we found high Ga-contents up to around 20 atomic-\%. At high $\mathrm{Ga}^{+}$ion doses, the Ga forms nanoparticles which could act as scattering centers. We expect both findings to strongly influence the performance of SiN phase plates. Further problematic are the interface widths between milled and unmilled regions, which are between 100 and $200 \mathrm{~nm}$. This far exceeds the beam envelope, and can be explained by a swelling of the $\mathrm{SiN}$ due to $\mathrm{Ga}$ incorporation. All of these factors need to be accounted for when preparing SiN phase plates, and we hope that our work will aid the community in preparing high-quality phase plates.

\section{References:}

[1] M Uchida and A. Tonomura, Nature 464 (2010), p. 737.

[2] C Ophus et al., Nature Communications 7 (2016), p. 10719.

[3] R Shiloh et al., Ultramicroscopy 189 (2018), p. 46.

[4] TR Harvey et al., New Journal of Physics 16 (2014), p. 09303.

[5] Work at the Molecular Foundry was supported by the Office of Science, Office of Basic Energy Sciences, of the U.S. Department of Energy under Contract No. DE-AC02-05CH11231. Daniel B. Durham was supported by STROBE: A National Science Foundation Science \& Technology Center under Grant No. DMR 1548924.

a)

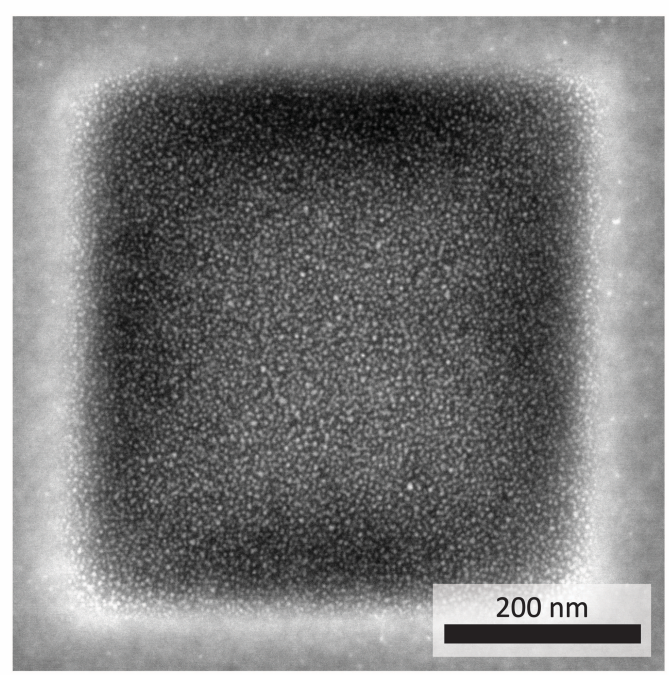

b)

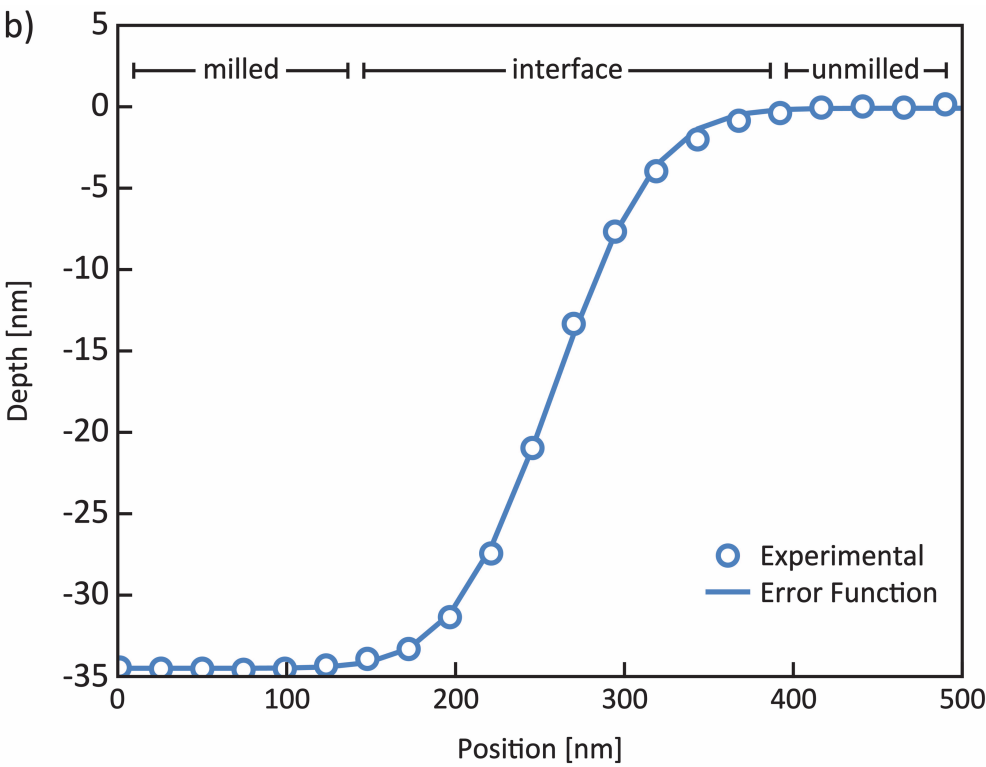

Figure 1. a) Squares such as the one shown in this STEM image were used to analyze the effects of the mill parameters. Nicely shown in the image are Ga nanoparticles which formed within the milled area. b) AFM measurements were used to determine the interface width between milled and unmilled areas. The interfaces can be described analytically using error functions. 